\title{
The inherent coupling of charge transfer and mass transport processes: the curious electrochemical reversibility
}

\author{
Renato Seeber ${ }^{1,2} \cdot$ Chiara Zanardi $^{1} \cdot$ György Inzelt $^{3}$
}

Received: 25 January 2016/Accepted: 1 May 2016/Published online: 18 May 2016

(C) Springer International Publishing Switzerland 2016

\begin{abstract}
As a complement to a previous contribution from us, the mass transport mechanisms of the electroactive species to and from the electrode in an uncomplicated electrode mechanism are considered. The electrode process as a whole is discussed, with emphasis to its reversibility degree, as results from the relevant responses in controlled potential techniques, such as chronoamperometry and current sampling voltammetry, linear sweep and cyclic voltammetry, and in rotating disk voltammetry. The electrode process as a whole, composed by charge transfer and mass transport steps that concur to condition the current flowing, is analysed on the basis of the relative rates of the two steps, as well of the time window within which the process is observed. The so-called 'boundary value problem' for uncomplicated charge transfers with different reversibility degrees is outlined. Supplementary Material is available, in which the simulated concentration profiles for reduced and oxidised species reacting at an electrode, at which a triangular potential waveform is applied, are linked to the corresponding current densities.
\end{abstract}

Electronic supplementary material The online version of this article (doi:10.1007/s40828-016-0027-3) contains supplementary material, which is available to authorized users.

Renato Seeber

renato.seeber@unimore.it

1 Department of Chemical and Geological Sciences, University of Modena and Reggio Emilia, Via G.Campi, 103, 41126 Modena, Italy

2 Institute for Organic Synthesis and Photoreactivity, National Council of Research (CNR), Research Area of Bologna, Via P. Gobetti 101, 40129 Bologna, Italy

3 Department of Physical Chemistry, Institute of Chemistry, Eötvös Loránd University, Pázmány Péter sétány 1/A, Budapest 1117, Hungary
Keywords Electrode reactions - Electrolytic cells · Mass transport mechanisms - Laws of diffusion - Conditional reversibility degree . Controlled potential techniques . Boundary value problem

\section{Introduction}

In previous contributions [1, 2], thermodynamics and kinetics have been treated 'separately' from the other steps of the whole charge-transfer process. However, whenever electric current crosses the 'electron conductorlelectrolyte' interface, the following issues need being considered: (1) How is the reactant transported to the interface and how does the product leave it, i.e. what manages the mass transport to and from the electrode surface? (2) How does the charge transfer at the interface interplay with the mass transport? (3) How do electrode thermodynamics, chargetransfer kinetics, and mass transport kinetics determine the overall process and condition the current responses accounting for its occurrence?

The link between thermodynamics and kinetics governing an electrochemical process is a crucial point dealt with in Ref. [2]. Though it was there discussed in an electrochemical frame, most of the points are common to physical and chemical events, as it will be tentatively illustrated hereafter. Moreover, in electrodics, the mass transport constitutes an additional kinetically controlled event that will be extensively discussed in the present contribution.

A first point to consider in the exam of a more or less complex process consists of the vague border between reversibility and non-reversibility, which was also in part discussed in Ref. [2]. Such a sort of grey area descends from the necessity to consider, together with the 
thermodynamic issue, also the kinetic ones. This implies to consider the intrinsic characteristics of the electrochemical system and experimental conditions that may drastically change the way that the process shows itself through the relevant electrochemical responses. This issue also emerges quite often throughout the present contribution, and for this reason, room should be dedicated to discuss it.

In the common language, the denomination 'reversible' is devoted to an event that can proceed in either of the two directions: both in the forward sense and in the backward sense. This is not, for instance, the case of the fall of a body or, in chemistry, of the oxidation of glucose by an oxidant to give $\mathrm{CO}_{2}$ and $\mathrm{H}_{2} \mathrm{O}$. The forward kinetic constant is substantially different from zero, while the backward kinetic constant is not: there is no way to go back, and the equilibrium constant of the reaction leading to the formation of the products assumes a huge value.

This simple definition is still valid in the experimental science, being, however, complemented by more subtle arguments in a thermodynamic frame. The correct definition of a (theoretically) reversible process is that it occurs through equilibrium states, i.e. it has to proceed through infinitesimal changes in the variables defining the evolving system [3]. The dramatic consequence is that in the real world, it could not proceed at all. A compromise is necessary, because the ideal reversibility is a condition that can only be approached in the real world.

The case of a galvanic cell working at different rates, illustrated in Ref. [2], represents a particularly nice example of progressive approach to reversibility in the thermodynamic meaning: an external load is suitable to modulate the rate of the spontaneous cell reaction, as well as the conversion of chemical (free) energy into useful work [3]. The higher the external resistance, the lower the current, and the slower the reaction, the "closer to reversibility' the spontaneous process occurring in the cell.

An example of different strong interactions between thermodynamics and kinetics is evident when considering the transformation of diamond to graphite. Diamond is a metastable allotropic form of carbon and is less stable than another form, i.e. graphite, so that the equilibrium constant is in favour of the formation of graphite. However, the conversion rate from diamond to graphite is too low under standard conditions that the reaction seems not to proceed at all: kinetics may render thermodynamics poorly meaningful, in practice. However, if we could observe the phenomenon over an extremely long time, actually very much longer than our own life, we could observe the progressive formation of graphite, until the equilibrium is achieved. An extraordinarily important experimental variable is introduced: the time window over which the event is observed.

\section{The electrode charge-transfer process}

\section{Deconvolving the process}

In previous contributions $[1,2]$, we dealt with the thermodynamic and kinetic aspects of redox reactions occurring either by direct contact of the reagents with each other or inside an electrochemical cell. Both the cases of a spontaneous process in a galvanic cell and of a forced one in an electrolytic cell were considered, especially focusing the attention to the oxidation or reduction half-reaction at a single electrode, i.e. the charge-transfer step or electrontransfer step.

An electrode reaction necessarily involves, together with the transfer of electrons, the presence of one or more charged species. It is an interfacial reaction, in which the energy of electrons and ions depends on the inner potential of the metal and solution close to it: such a difference is identifiable with the electrode potential, $E$.

In an electrolytic cell, the potential difference between two electrodes can conveniently be varied to regulate the reaction rate at the electrode of interest, i.e. at the so-called working electrode (WE). The cell reaction occurs at two electrodes, and the electric current flows through the cell (inner circuit) and through the outer conductor connecting the two electrodes (outer circuit). The second electrode is the so-called auxiliary electrode. In the three-electrode arrangement (three-electrode cell), a reference electrode (RE) is also present, allowing the best control of the potential of the WE [1]. Therefore, we will focus on the events occurring at a single electrode.

In principle, the electrode reaction involves all the steps (chemical reactions, structural reorganizations, and adsorptions) accompanying the charge transfer. However, only this last step is considered by the mathematical expressions of the current density as a function of the electrode potential, namely, the Erdey-Grúz Volmer (EGV) equation, described in Ref. [2]. On the other hand, even when considering the simplest electrode process, the mass transport of the species involved has to be considered as a necessary complement to the charge transfer. Diffusion, migration, and convection are the three possible mass transport processes. Diffusion should always be considered, because as the reagent is consumed and the product is formed at the electrode, concentration gradients between the vicinity of the electrode and the bulk of the solution ${ }^{1}$ arise: the reactant species move towards the electrode surface, and product species leave the interfacial region

\footnotetext{
1 With the term 'bulk of the solution', we refer to whatever solution volume in which the concentrations of the electroactive species are unchanged with respect to the initial values, independently of what happens at the electrode.
} 
(interphase). ${ }^{2}$ Similarly, migration, i.e. mass transport due to an inner potential gradient in the solution near the electrode, should also be considered. The charge transfer, in fact, necessarily alters the charge balance of the chemical species close to the electrode. As illustrated afterwards, a specific modification of the solution is made in order that transport by migration is minimised.

Once considering the diffusion and charge-transfer steps in series to each other, it should be recalled that the rate of a stepwise event in which the slowest step is the first one only depends on the rate of this; in the opposite case, the rates of all steps preceding the slowest one concur to define the overall rate. Hence, whenever the kinetics of the charge transfer is limiting, the rate at which the electroactive species is made available at the electrode should also be considered.

We will deal here with the phenomena occurring in the solution as a consequence of depletion and production of electroactive species at the electrode: the arising of a mass gradient at any points between the electrode surface and the bulk of the solution. We will couple this event to the charge-transfer step, in the frame of an overall unique electrode process: the events of charge transfer and mass transport by diffusion are concomitant, and affect each other: the charge-transfer step induces mass transport and mass transport support charge transfer. The link between the two steps of the electrode process constitutes a key point of what discussed here.

The subjects treated in the present contribution hold in all situations typical of potentiostatic, galvanostatic, or potentiodynamic techniques, in which a constant or programmed perturbation of the potential or of the current at WE is applied. In practice, the most widespread cases are: (1) chronoamperometric technique, in which the current is measured as a function of time, WE being polarised at a constant potential after a potential step perturbation; (2) voltammetric techniques, in which the current is measured at WE polarised at potentials varying during time; (3) galvanostatic techniques, in which a constant current is imposed to the WE and the potential correspondingly assumed is measured at passing time. In cases (1) and (2), the so-called potential waveform, $E(t)$, gives account of the kind of the technique used and of the specific relevant parameters.

The most widely used controlled potential techniques, beside the chronoamperometry and to the strictly related voltammetric techniques, i.e. current sampling voltammetry (CSV), are linear sweep and cyclic voltammetry

\footnotetext{
${ }^{2}$ For the sake of simplicity, throughout the whole article, we will simply call electrode the interphase directly involved in the electron transfer. In fact, it is the electron conducting phase of the electrode (metal, carbon together with their surface modification, e.g. electron conducting oxide), as generally used in laboratory practice.
}

(LSV and $\mathrm{CV}$, respectively), and rotating disc electrode (RDE) voltammetry. A short treatment of these techniques is made in the following, in order that the reader may have an idea of how the equations and the arguments exposed here and in Ref. [3] give account of actual experimental responses. The exhaustive treatment of the individual electrochemical techniques is beyond the scope of the present contribution; it can be found in numerous excellent textbooks [4-10].

\section{Equilibrium conditions in the absence and presence of current flow}

Different models of the so-called electrical double layers have been developed to account for the composition of the solution close to a charged metal surface, in terms of excess of ionic and non-ionic species, in the absence of faradic current. Figure 1 shows a sketch of the denominations and characteristics of the layers of solution constituting the electrical double layer. The solution layer closest to the metal, which is either spontaneously charged or forced to assume a given potential, contains solvent molecules and, sometimes, other ions or molecules that are specifically adsorbed (compact, Helmholtz, or Stern layer). In particular, the region containing electrical centres of the adsorbed ions constitutes the inner Helmholtz plane (IHP), whereas the locus of centres of the solvated ions nearest to the metal is the outer Helmholtz plane (OHP). Long-range electrostatic forces exert influence on ions in solution; the effect is essentially independent of the chemical properties of the species. These are distributed in a region called the

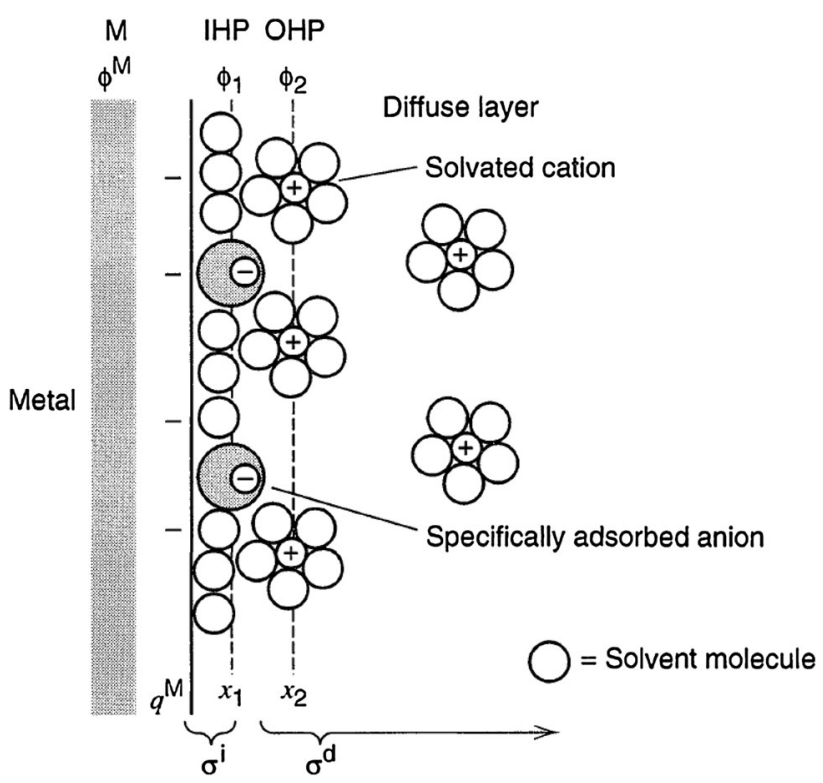

Fig. 1 Electrical double layer. Reprinted with permission from Ref. [8] 
diffuse layer, which extends from the OHP into the bulk of the solution.

Once the electrode is polarised, i.e. assumes a potential different from that at the equilibrium, the extent of the overall current measured in a WE-AE circuit is given by the sum of two components: (1) the first one is due to electrons that do cross the electrodelsolution interface for the occurrence of the charge transfer: it constitutes the socalled faradic current, namely, the current considered here; (2) the second one does not imply that electrons pass from the electrode to the solution or vice versa and is due to charging-discharging of the electrical double layer, similar to the charging-discharging of a capacitor: it is the socalled capacitive current or, more precisely, differential capacitive current. This capacitive component of the overall current most often constitutes a 'noise' in the frame of the characterisation or quantification of an electroactive species in solution.

To deal with a charge-transfer step, kinetic parameters, namely, the standard kinetic rate constant of the heterogeneous charge transfer $\left(k_{\mathrm{s}, \mathrm{h}}\right)$ and the exchange current density $\left(j_{0}\right)$, have been defined [2]. They represent the kinetic constant of both forward and backward redox reactions at $E=E^{\circ \prime}$ and the exchange current density, i.e. the current density flowing with equal intensity in both the anodic and cathodic directions at the equilibrium potential, $E=E_{\text {eq }}$, respectively. In principle, an infinite rate of the charge transfer is required to define a process reversible in character. What introduced in the present contribution, namely, the mass transport, constitutes an additional kinetic factor to consider with respect to the reversibility degree exhibited by a charge-transfer step. The different situations arising from the necessity to consider thermodynamic and all kinetic factors will be examined in the following.

In any cases, reversibility requires that the Nernst equation can be applied once expressed as a function of the concentrations of the reacting species at the closest approach to the electrode: equilibrium conditions are achieved, and the Nernst equation is obeyed, in spite of the flowing current. However, the upsurge of a conflict between the experimental limits and the theoretical idea of 'infinite rate' is evident. It is, hence, easy to accept a priori the assumption that a charge transfer appears to be reversible when its rate is above a certain limit, making the Nernst equation suitable to account for the values of the concentrations at the surface of the electrode.

As briefly introduced in the beginning of this study, the reversibility degree of a charge transfer, as inferred from the relevant electrochemical responses, is only meaningful once connected to the experimental conditions under which the response is observed. It is proper to speak of a conditional (or apparent) reversibility degree, observed by applying a given technique and under given experimental conditions, with particular emphasis to (1) the mass transfer to the electrode and (2) the time window of observation. This means that a process, on the basis of the relevant responses, may appear as a reversible or as a nonreversible one, when adopting different conditions to look at it. It follows that the quantities defining the kinetics, such as $k_{\mathrm{s}, \mathrm{h}}$ and $\alpha$ (the charge transfer coefficient) or $j_{0}$, may be computed only when they are not too high, i.e. from experimental responses recorded under conditions in which the charge transfer exhibits non-reversible behaviour.

In a pure kinetic frame, the charge-transfer step is only considered: it is said to be irreversible when it is very sluggish $\left(k_{\mathrm{s}, \mathrm{h}}\right.$ and $j_{0}$ are very small). In this case, the anodic charge-transfer kinetics and the cathodic charge-transfer kinetics are never simultaneously significant. To observe a current, the charge-transfer reaction has to be strongly activated either in the cathodic or in the anodic direction by application of a suitable overpotential; the charge transfer in the opposite direction does not occur at all. When the electrode process is neither very facile nor very sluggish, we speak of quasi-reversible behaviour.

The contemporary dependence of the conditional reversibility degree on the parameters of the charge transfer and of the mass transport rates, as well on the time window, is considered in Fig. $2 . k_{\mathrm{m}, \mathrm{Red}}=D_{\mathrm{Red}} / \delta_{\mathrm{Red}}$ is the mass transport coefficient, where $D_{\text {Red }}$ is the diffusion coefficient of reactant species Red [see Eq. (1)] and $\delta_{\text {Red }}$ is the diffusion layer thickness (see later); a parameter, $\tau$, accounts for the time window of the experiment.

Making reference to Ref. [2], let us recall a few equations accounting for the kinetics of a generic oxidation electrode process:

$\mathrm{Red} \leftrightarrows \mathrm{Ox}+\mathrm{ne}$

Recalling that a one-electron charge transfer constitutes invariably the rate-determining step of the electron transfer, the current density may be expressed in two forms. The first one is

$j(t)=j_{0}(t)\left\{\frac{c_{\text {Red }}(0 ; t)}{c_{\text {Red }}^{*}} \exp \left[\frac{\alpha F \eta}{R T}\right]-\frac{c_{\text {Ox }}(0 ; t)}{c_{\text {Ox }}^{*}} \exp \left[\frac{-(1-\alpha) F \eta}{R T}\right]\right\}$

where $\eta$ is the charge transfer overvoltage and indicates the difference between the imposed and the equilibrium potential. Alternatively, Eq. (2) can be written in the form:

$$
\begin{aligned}
j(t)= & n F k_{\mathrm{s}, \mathrm{h}}\left\{c_{\text {Red }}(0 ; t) \exp \left[\frac{\alpha F\left(E-E^{0^{\prime}}\right)}{R T}\right]\right. \\
& \left.-c_{\text {Ox }}(0 ; t) \exp \left[\frac{-(1-\alpha) F\left(E-E^{0^{\prime}}\right)}{R T}\right]\right\}
\end{aligned}
$$




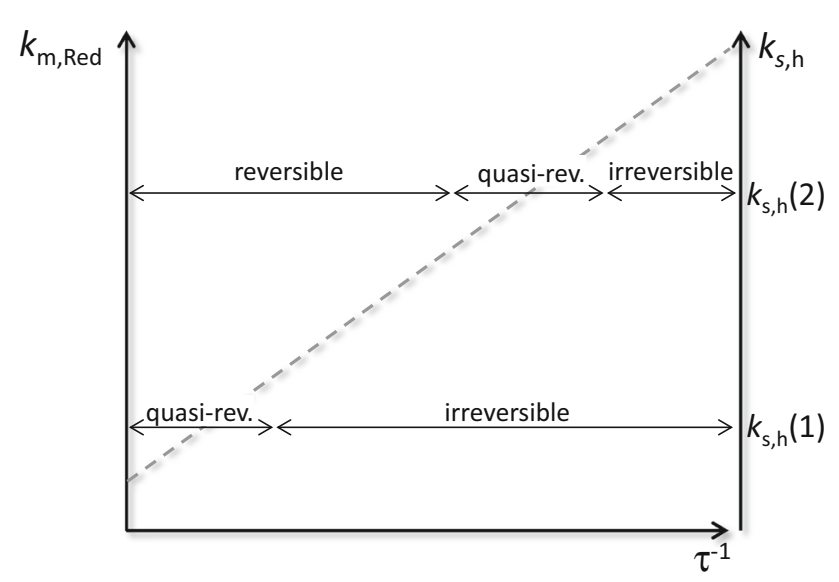

Fig. 2 Illustration of the conditional reversibility degree of charge transfers as a function of the relevant intrinsic characteristics, of the diffusion rate, and of the time window of the experiment, $\tau$. Two systems with different electron-transfer rates $\left[k_{\mathrm{s}, \mathrm{h}}(1)\right.$ and $\left.k_{\mathrm{s}, \mathrm{h}}(2)\right]$ are considered. The standard rate constants $\left[k_{\mathrm{s}, \mathrm{h}}(1)\right.$ and $\left.k_{\mathrm{s}, \mathrm{h}}(2)\right]$ are the characteristics of the charge transfer kinetics of two exemplificative systems and $k_{\mathrm{m}, \text { Red }}$ accounts for the diffusion rate for the reduced reactant species [see Eq. (1)] in solution. If $k_{\mathrm{s}, \mathrm{h}} \gg k_{\mathrm{m}, \text { Red }}$, the system appears as a reversible one, while in the case of $k_{\mathrm{m}, \operatorname{Red}} \gg k_{\mathrm{s}, \mathrm{h}}$, irreversible behaviour is observed. Adapted from Ref. [11]

where $E^{0 \prime}$ is the formal potential of the Ox/Red couple.

It is easy to see that for $\eta=0$, i.e. at the equilibrium potential $\left(E=E_{\text {eq }}\right), j(t)=0$, so that

$c_{\text {Red }}(0 ; t)=c_{\text {Red }}^{*}$

and

$c_{\mathrm{Ox}}(0 ; t)=c_{\mathrm{Ox}}^{*}$

where $c_{\mathrm{Ox}}^{*}$ and $c_{\mathrm{Red}}^{*}$ are the concentrations of Red and $\mathrm{Ox}$ species, respectively, in the bulk of the solution, whereas $c_{\mathrm{Ox}}(0 ; t)$ and $\mathrm{c}_{\mathrm{Red}}(0 ; t)$ are the relevant concentrations at the electrode surface. On the other hand, according to Eq. (2), $j(\mathrm{t})$ is modulated by $j_{0}(\mathrm{t})$, i.e. the current density that 'flows' with equal intensity in either directions at $E=E_{\mathrm{eq}}$. Assuming $c_{\mathrm{Red}}^{*}=c_{\mathrm{Ox}}^{*}$, it follows that $E_{\mathrm{eq}} \equiv E_{\mathrm{Ox} / \operatorname{Red}}^{0^{\prime}}$, so that Eq. (3) may be written under the form:

$$
\begin{aligned}
j_{0}(t) & =n F k_{\mathrm{s}, \mathrm{h}} c_{\mathrm{Red}}(0 ; t) \exp \left[\frac{\alpha F\left(E_{\mathrm{eq}}-E^{0^{\prime}}\right)}{R T}\right] \\
& =n F k_{\mathrm{s}, \mathrm{h}} c_{\mathrm{Ox}}(0 ; t) \exp \left[\frac{-(1-\alpha) F\left(E_{\mathrm{eq}}-E^{0^{\prime}}\right)}{R T}\right]
\end{aligned}
$$

Alternatively, being $j_{0}$ defined in equilibrium conditions:

$$
\begin{aligned}
j_{0} & =n F k_{\mathrm{s}, \mathrm{h}} c_{\mathrm{Red}}^{*} \exp \left[\frac{\alpha F\left(E_{\mathrm{eq}}-E^{0^{\prime}}\right)}{R T}\right] \\
& =n F k_{\mathrm{s}, \mathrm{h}} c_{\mathrm{Ox}}^{*} \exp \left[\frac{-(1-\alpha) F\left(E_{\mathrm{eq}}-E^{0^{\prime}}\right)}{R T}\right]
\end{aligned}
$$

In view of Eqs. (4) and (5):
$E_{\mathrm{eq}}=E_{\mathrm{Ox} / \mathrm{Red}}^{0^{\prime}}+\frac{R T}{n F} \ln \frac{c_{\mathrm{Ox}}^{*}}{c_{\mathrm{Red}}^{*}}$

By a number of substitutions and suitable elaborations, Eq. (7) may be rewritten under the form:

$j_{0}=n F k_{\mathrm{s}, \mathrm{h}}\left[c_{\mathrm{Red}}^{* \alpha} c_{\mathrm{Ox}}^{*-(1-\alpha)}\right]$

The ratio between Eqs. (3) and (9) is hence:

$$
\begin{aligned}
\frac{j(t)}{j_{0}} & =\frac{n F k_{\mathrm{s}, \mathrm{h}}\left\{c_{\mathrm{Red}}(0 ; t) \exp \left[\alpha F\left(E-E^{0 \prime}\right) / R T\right]-c_{\mathrm{Ox}}(0 ; t) \exp \left[-(1-\alpha) F\left(E-E^{0 \prime}\right) / R T\right]\right\}}{n F k_{\mathrm{s}, \mathrm{h}}\left[c_{\mathrm{Red}}^{* \alpha} c_{\mathrm{Ox}}^{*-(1-\alpha)}\right]} \\
& =\frac{c_{\mathrm{Red}}(0 ; t) \exp \left[\alpha F\left(E-E^{0 \prime}\right) / R T\right]-c_{\mathrm{Ox}}(0 ; t) \exp \left[-(1-\alpha) F\left(E-E^{0 \prime}\right) / R T\right]}{c_{\mathrm{Red}}^{* \alpha} c_{\mathrm{Ox}}^{*-(1-\alpha)}} \\
& =\frac{c_{\mathrm{Red}}(0 ; t) \exp \left[\alpha F\left(E-E^{0 \prime}\right) / R T\right]}{c_{\mathrm{Red}}^{*}}-\frac{c_{\mathrm{Ox}}(0 ; t) \exp \left[-(1-\alpha) F\left(E-E^{0 \prime}\right) / R T\right]}{c_{\mathrm{Ox}}^{*}}
\end{aligned}
$$

Figure 3 shows $j$ vs. $\left(E-E_{\mathrm{eq}}\right)$, being $E_{\mathrm{eq}} \equiv \mathrm{E}^{0 \prime}$ under the assumed conditions, i.e. $j(t)$ vs. $\eta$ curves computed by Eq. (10), for a given $j_{0}$ value, i.e. for a given reversibility degree of the charge transfer. The voltammetric curves consist of both an anodic and a cathodic portion. The further assumption that $\alpha=0.5$ implies that $j(t)$ is an antisymmetric function of the $t$ variable, i.e. of the linearly related $E$ variable.

Equation (10) is the current-overpotential equation. As a version of the EGV equation, it expresses the actual density current that considers for the concentration values of the electroactive species at the electrode that are different from those in the bulk of the solution. The finite rate at which Red and Ox species are supplied to and subtracted from the electrode surface, respectively, is considered, despite the mechanisms through which mass transport is operative are not explicitly dealt with. The corresponding effects are evidenced by Eq. (10): the concentration values at the electrode are predicted by the Nernst equation in the case of a reversible charge transfer, and for non-reversible processes, they can be computed once $k_{\mathrm{s}, \mathrm{h}}$ and $\alpha$, or $j_{0}$, are known. $j(t)$ is then computed by Eq. (2), and consequently, $c_{\mathrm{Red}}(0 ; t)$ and $c_{\mathrm{Ox}}(0$; $t$ ) are drawn out by Eq. (3). According to Eq. (10), this means that, at a given value of $j_{0}$, the lower $c_{\operatorname{Red}}(0 ; t)$ or and the higher $c_{\mathrm{Ox}}(0 ; t)$ to realise the higher has to be the overpotential to impose to the electrode. On the other hand, $j(t)$ increases at increasing $j_{0}$ for each overvoltage value, $c_{\mathrm{Ox}}(0 ; t)$ and $c_{\mathrm{Red}}(0 ; t)$ being fixed. The plateau values of the anodic and cathodic currents are defined as the limiting currents for anodic oxidation and cathodic reduction; they correspond to the overvoltages high enough to make $c_{\text {Red }}(0$; $t)=0$ or $c_{\mathrm{Ox}}(0 ; t)=0$, respectively.

\section{The electrode process as a whole: charge transfer plus mass transport}

The most physically perceivable approach to the calculation of current/time or current/potential curves is quite 
Fig. 3 Current-overpotential curve for an uncomplicated electrochemical oxidation with $c_{\mathrm{Red}}^{*}=c_{\mathrm{Ox}}^{*}$ and $\alpha=0.5$ :

$E_{\text {eq }} \equiv E^{0 \prime}$. The dotted lines represent the anodic and the cathodic components, whereas the solid line represents the total current. $j_{\mathrm{L}}$ and $-j_{\mathrm{L}}$ are the limiting currents at sufficiently positive and negative potentials, respectively. $j_{0}=0.2 j_{\mathrm{L}}$, which fixes a ratio between the charge transfer and the diffusion rates. Every $j$, except for $j_{0}$, stands for $j(t)$. Adapted from Ref. [8]

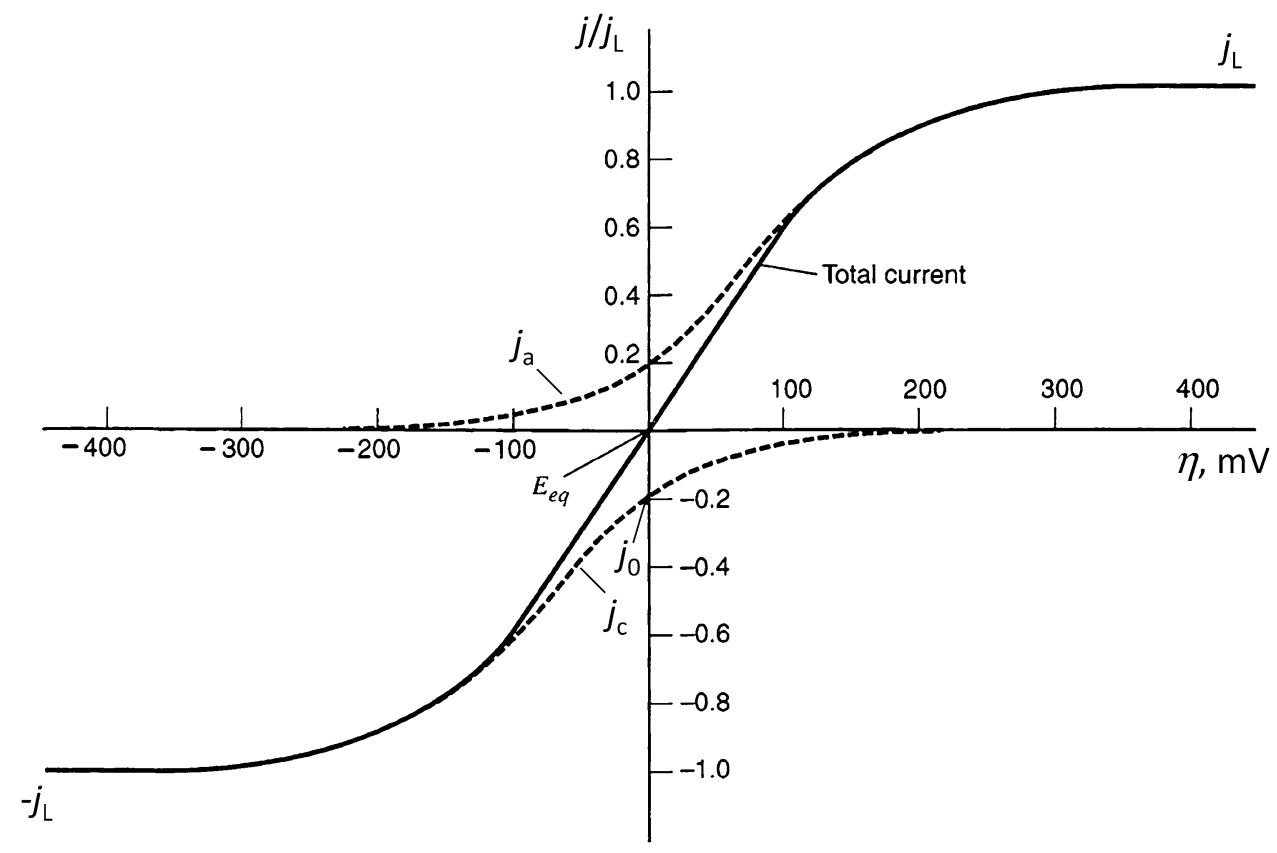

different with respect to that followed to draw out Eq. (10). Figure 4 shows the sequence of steps of an uncomplicated charge transfer, i.e. a process not involving either chemical reactions or adsorption events in charge of reactants or products, which are both soluble in the solution phase. Considering the sequence of steps constituting the electrode process as a whole, the charge transfer at the electrode clearly acts as the primary source of the perturbation. Actually, the events at the electrodelsolution interface are often complex; in series or in parallel to more or less reversible charge transfer(s), homogeneous reactions and weak or strong adsorptions may take place in complex mechanisms. The fitting with theoretical responses or with calculated trends of typical quantities of the responses is of help. In particular, the changes occurring at the electrodelsolution interface, e.g. adsorptions, are often defined by proper fitting with the responses of the so-called equivalent circuits. ${ }^{3}$

\footnotetext{
${ }^{3}$ So-called equivalent circuits consist of suitable resistances, capacitances, and even inductances, both in series and in parallel to each other. They are typically drawn to give reason for the behaviour of an electrodelsolution system in the so-called impedance or faradic impedance technique, as deduced from measurements performed, at a constant d.c. potential, by applying alternating voltages at different frequencies: the relevant in-phase and out-of-phase current components are analysed. By fitting modulus and phase of the experimental current to those computed for a given equivalent circuit, precious information about the structure of the electrodelsolution double layer, crossed or not by electrons, may be gained. In measurements at controlled potential, i.e. in continuous current amperometric techniques, the relationship between the experimental responses and the relevant equivalent circuits is very much more labile. However, invoking a sort of equivalent circuit at a qualitative level may be helpful in similar cases as well.
}

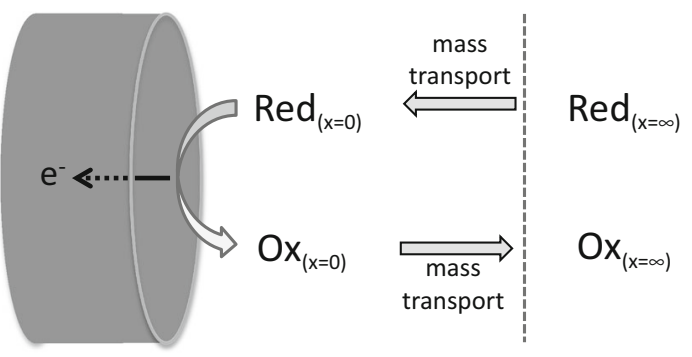

Fig. 4 Scheme of an uncomplicated charge transfer

As discussed above, the occurrence of the reaction in Eq. (1) causes inhomogeneity in the solution, as to both composition and electric charge: gradients different from zero arise for both Ox and Red concentrations, as well as for electric potential.

\section{The mass transport}

The general formula accounting for all mechanisms of mass transport for the $i$ species along any directions is given by the following general equation:

$J_{i}=-D_{i} \nabla c_{i}-\frac{z_{i} F}{R T} D_{i} c_{i} \nabla+\bar{v} c_{i}$

where $J_{i}$ represents the flux of the transferring species $i$, i.e. the number of moles through a unitary surface area in a unitary time interval, expressed in mol $\mathrm{cm}^{-2} \mathrm{~s}^{-1} ; D_{i}$ is the diffusion coefficient of the $i$ th species, in $\mathrm{cm}^{2} \mathrm{~s}^{-1} ; z_{i}$ is the charge of the ion considered; $c_{\mathrm{i}}$ is the concentration of the $i$ th species, expressed in $\mathrm{mol} \mathrm{cm} \mathrm{cm}^{-3} ; \bar{v}$ is the vectorial sum 
of the fluid velocity in the three Cartesian coordinates: $v_{x}+v_{y}+v_{z}$; and $\nabla$ is the divergence vector operator or Laplace operator $\left(=\frac{\partial}{\partial x}+\frac{\partial}{\partial y}+\frac{\partial}{\partial z}\right.$ in a system of the three Cartesian coordinates), accounting for the vectorial sum of the concentration or inner potential gradients, in the first and second terms of Eq. (11), respectively.

The concentration gradients of both reactant and product induced by the electrode charge-transfer forces their diffusion toward and away from the electrode surface, to pursue homogeneity of the solution. On the other hand, the inner potential gradient, $\nabla$, forces the charged species to migrate: in the case of the oxidation reaction expressed by Eq. (1), migration of anionic species toward and of cationic species away from the proximity of the electrode occur. Actually, in controlled potential amperometric techniques, an inert salt, also called supporting electrolyte, is deliberately added to the solution to compensate the unbalance of electric charges for the very most part. The additional basic role of the supporting electrolyte is to lower the solution resistance as much as possible. To achieve these goals, it is added to the solution in a large excess with respect to the electroactive species. The denomination 'inert salt' is due to the requirement not to alter the nature of the species in solution, as well to be resistant with respect to anodic oxidation or cathodic reduction as much as possible. This property widens the so-called potential window of the solution, i.e. the potential range within which electrochemical processes due to solvent or to inert salt oxidation or reduction do not take place, allowing there the detection of species of interest (see Fig. 5).

As a support to homogenisation of the solution, hence, to diffusion, solution stirring or electrode movement with respect to the solution may be applied: in this case, convection also concurs to mass transport. However, precise control of such a mass transport mechanism can be

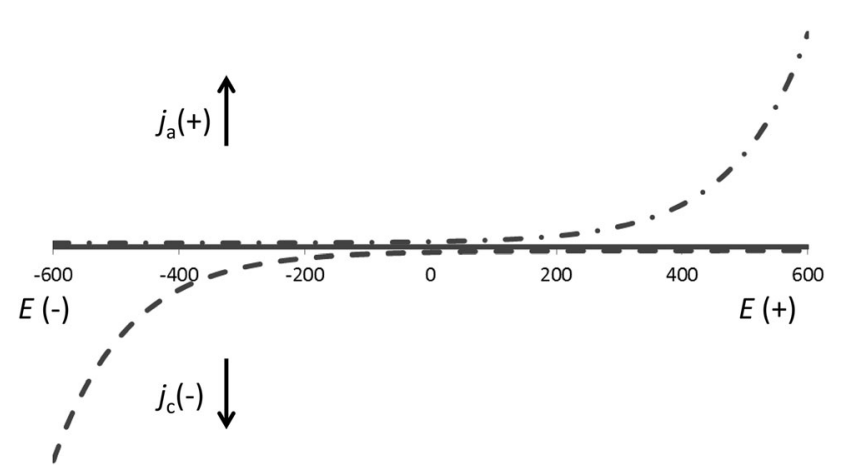

Fig. 5 Current vs. potential plots in the presence of a given solvent and an inert salt in solution. Three possible situations are illustrated: (1) solvent and supporting electrolyte are not electroactive at all (solid line); (2) solvent or supporting electrolyte is oxidised at the anode (dash-dotted line); (3) solvent or supporting electrolyte is reduced at the cathode (dashed line) achieved using an RDE; reproducibility is possible with such a setup, and the hydrodynamic equations have been solved in this case. On the other hand, in the most widely used diffusion controlled potential techniques, e.g. chronoamperometry, linear sweep and cyclic voltammetry, or pulse techniques, convection is absent and carefully prevented from incidentally occurring. Diffusion plays, hence, the role of mass transfer process.

\section{Diffusion}

What described above suggests privileging the treatment of one among the mass transport mechanisms, namely, diffusion. Experimental situations are often realised in order that semi-infinite linear diffusion condition is well approximated: the flux of species to a planar electrode surface occurs orthogonal to the diffusion direction. This process allows the easy computation of the current density. Strictly speaking, semi-infinite linear diffusion conditions require a perfectly planar electrode surface of infinite area, the solution extending at infinite distance from it. Under similar conditions, the concentration gradient of the diffusing species is only different from zero along the direction orthogonal to the electrode surface, and the perturbation arising at the electrode is allowed to extend away from it without meeting with any physical boundary. In the frame of a Cartesian system in which the $x$ axis is orthogonal to the electrode surface, the following relationships hold:

$$
\begin{aligned}
& \frac{\partial c_{\text {Red }}(x ; y ; z ; t)}{\partial y}=0 \\
& \frac{\partial c_{\text {Ox }}(x ; y ; z ; t)}{\partial y}=0 \\
& \frac{\partial c_{\text {Red }}(x ; y ; z ; t)}{\partial z}=0 \\
& \frac{\partial c_{\text {Ox }}(x ; y ; z ; t)}{\partial z}=0
\end{aligned}
$$

On the other hand, it holds that:

$$
\begin{aligned}
& \lim _{x \rightarrow \infty} c_{\text {Red }}(x ; y ; z ; t)=c_{\mathrm{Red}}^{*} \\
& \lim _{x \rightarrow \infty} c_{\text {Ox }}(x ; y ; z ; t)=c_{\mathrm{Ox}}^{*}
\end{aligned}
$$

The conditions required by the semi-infinite linear diffusion are seemingly impossible to realise in an experimental frame. Actually, experimental conditions are well possible in which the responses differ from those computed under the theoretical model to a negligible extent, actually much less than the experimental uncertainty. In the case of a disk electrode, a diameter of 3 or $4 \mathrm{~mm}$ is high enough to make the diffusion component along the directions parallel to the electrode surface negligible, satisfying, hence, Eqs. (12-15). 
Furthermore, if the walls of the electrochemical cell, which constitute the obvious physical boundary to diffusion, are located a few micrometres far from the electrode surface, $c_{\text {Red }}$ and $c_{\text {Ox }}$ at such a distance are meaninglessly different from $c_{\mathrm{Red}}^{*}$ and $c_{\mathrm{Ox}}^{*}$, respectively. Under conditions in which $x$ axis constitutes the only diffusion direction, the $c_{\text {Red }}$ Vs. $x$ and $c_{\text {Ox }}$ vs. $x$ plots are called the concentration profiles of Red and Ox species, respectively.

In this respect, let us consider that the most dramatic effect of the charge transfer at the electrode on the concentration values clearly occurs when a sudden change in the electrode potential is applied, suitable to 'instantaneously' force to zero the concentration of electroactive species at the distance of closest approach. The following equation expresses the concentration profile for Red species in similar conditions:

$c_{\text {Red }}(x ; t)=c_{\text {Red }}^{*} \operatorname{erf}\left[\frac{x}{2\left(D_{\text {Red }} t\right)^{1 / 2}}\right]$

where erf is the so-called error function. The values of the error function rise very rapidly toward the unitary asymptotic value: when the argument of erf holds 1,2, and 3,i.e. when $x$ is 2 , 4, and 6 times the expression $\sqrt{D_{\mathrm{Red}} t}$, the ratio $c_{\text {Red }}(x ; t) / c_{\text {Red }}^{*}$ assumes values of 0.84, 0.995, and 0.99998, respectively. As a consequence, the diffusion layer thickness, within which the concentrations of the electroactive species are significantly different from those in the bulk of the solution, is quite reasonably limited by a distance of $6 \sqrt{D t} \mathrm{~cm}$. A typical value of $D$ for small molecules in liquids at room temperature is ca. $10^{-6} \mathrm{~cm}^{2} \mathrm{~s}^{-1}$; a potential step imposing $c(x=0 ; t)=0$ for $t>0$, only involves a $0.002 \%$ concentrations change within a layer of ca. $60 \mu \mathrm{m}$, at $1 \mathrm{~s}$ polarisation, which is commonly a long enough time: a $60-\mu \mathrm{m}$-thick layer is wide enough to describe semi-infinite diffusion.

The diffusion to and from the electrode follows the equations developed long time ago for the diffusion of heat: Fick's first and second laws of diffusion. However, the boundary conditions under which the corresponding system of differential equations is solved are peculiar for the situations examined here.

The expression of Fick's first law of diffusion is very simple in the case of semi-infinite linear diffusion along the $x$ axis; as above discussed, only $\left(\frac{\partial c_{\operatorname{Red}}(x ; t)}{\partial x}\right)$ and $\left(\frac{\partial c_{\mathrm{Ox}}(x ; t)}{\partial x}\right)$ are different from 0 :

$J_{\operatorname{Red}}(x ; t)=-D_{\operatorname{Red}}\left(\frac{\partial c_{\operatorname{Red}}(x ; t)}{\partial x}\right)$

$J_{\mathrm{Ox}}(x ; t)=D_{\mathrm{Ox}}\left(\frac{\partial c_{\mathrm{Ox}}(x ; t)}{\partial x}\right)$

where $J_{\text {Red }}$ and $J_{\text {Ox }}$ account for the flux of the reduced and oxidised species, respectively, at an $x$ distance from the electrode. The reliability of the algebraic signs in Eqs. (18, 19 ) is clear by recalling that, in the present case of an anodic oxidation, the concentration gradient is positive for Red and negative for Ox species, respectively.

The flux of electroactive species at the electrode, i.e. at $x=0$, leads to the current density, $j(t)$, by transforming mol s${ }^{-1}$ into $\mathrm{C} \mathrm{s}^{-1}$ :

$j(t)=n F D_{\operatorname{Red}}\left(\frac{\partial c_{\text {Red }}(0 ; t)}{\partial x}\right)=-n F D_{\mathrm{Ox}}\left(\frac{\partial c_{\mathrm{Ox}}(0 ; t)}{\partial x}\right)$

According to Eq. (21), the current results positive in sign for the oxidation reaction considered here and negative for an eventual reduction.

For semi-infinite linear diffusion, the following form of Fick's second law expresses the change in concentration in any points of the solution as a function of time: at any $x$ distance from the electrode, it takes simply into account for the difference between the flux into and the flux out from a volume possessing $\mathrm{d} x$ width.

$$
\begin{aligned}
& \left(\frac{\partial c_{\text {Red }}(x ; t)}{\partial t}\right)=D_{\text {Red }}\left(\frac{\partial^{2} c_{\text {Red }}(x ; t)}{\partial x^{2}}\right) \\
& \left(\frac{\partial c_{\text {Ox }}(x ; t)}{\partial t}\right)=D_{\text {Ox }}\left(\frac{\partial^{2} c_{\text {Ox }}(x ; t)}{\partial x^{2}}\right)
\end{aligned}
$$

More complex expressions account for regimes different from semi-infinite diffusion, such as those that are operative at a mercury drop electrode, at microelectrodes, at porous electrodes, and so on.

\section{Mass transport coupled to charge transfer}

Mass transport and charge-transfer processes interplay with each other in determining the conditional reversibility degree of a charge-transfer process. As far as the Nernst equation is obeyed, whatever electrochemical technique is used to look at the Ox/Red system, the relevant responses are typical of a reversible charge transfer. As already evidenced, in fact, only a certain degree of reversibility is what makes sense in the real world: ideal reversibility requires infinite rate in the achievement of equilibrium, implying that the electrochemical activation free energy at $E=E^{0}$ should be equal to zero for both forward and backward reactions [2]. Apart from this limiting case, the faster the achievement of the equilibrium between electrode and redox system immediately close to it, the 'more reversible' the electrode-redox couple system.

By considering quite an extreme situation, in the case of potentiometric measurements, no current is involved, so that the solution is homogeneous at any distance from the indicator electrode: $c_{\text {Red }}(0 ; t)=c_{\text {Red }}^{*}$ and $c_{\mathrm{Ox}}(0 ; t)=c_{\mathrm{Ox}}^{*}$. The potential of the electrode is given such a long time to assume 
the value predicted by the Nernst equation that not so few redox systems, in which both the oxidised and the reduced forms of the redox couple are stable, and appear to behave reversibly. In this case, reversible behaviour implies the assumption by the electrode of the potential value that can theoretically be computed by the Nernst equation on the basis of $c_{\mathrm{Red}}^{*} / c_{\mathrm{Ox}}^{*}$ ratio. In other words, in potentiometry, once the concentrations of $\mathrm{Ox}$ and Red are fixed, we require $E$ to assume the value predicted by Eq. (8).

On the other hand, whenever the electrode is polarised at a given $E$ value, as it is the case of all controlled potential techniques, the ratio $c_{\mathrm{Ox}}(0 ; t) / c_{\text {Red }}(0 ; t)$ must assume the value predicted by the Nernst equation. When the potential is changed, either continuously or by subsequent steps, less time is given to the electrode-solution system to reach equilibrium, with respect to potentiometry. In conclusion, a redox system may appear reversible when 'observed' with a given technique, e.g. in potentiometry, or under given experimental conditions, e.g. when the potential applied to the electrode varies slowly, though appearing non-reversible when the potential is changed at higher rates. This means that, in the case of a controlled potential technique, reversibility is achieved under the condition that modifications of the potential causes novel concentrations at the electrode that are in agreement with the Nernst equation: more and more rapid the potential change, shorter and shorter the time window, faster and faster the change of the concentrations of the electroactive species at the surface has to be, in order that the response accounts for the occurrence of a reversible process. Thermodynamics may not be obeyed anymore: kinetic arguments concur to condition the current flowing, i.e. the rate of conversion of one to the other partner of the redox couple, namely, of Red to Ox in the example considered here.

The strict interplay between intrinsic reversibility of a charge transfer, as directly accounted for by $j_{0}$ or $k_{\mathrm{s}, \mathrm{h}}$, the time window on which the process is tested and the speed at which the mass transport of electroactive species occurs are of basic importance in conditioning the conditional degree of reversibility exhibited by an electrochemical response, as discussed with respect to Fig. 2.

As already underlined, mass transport and charge transfer can be considered as two processes in series: mass transport should necessarily supply the electroactive species that undergoes charge transfer. The current only depends on the rate of diffusion if it is the slower (more hindered) one, while the rates of both steps condition the overall rate in the case of a slower second step. It follows that for reversible charge-transfer processes, the diffusion is always the slower step: at any polarisation potential, the rate of the process is only conditioned by diffusion. This implies that, whenever the charge-transfer rate is high enough with respect to diffusion, the responses exhibit the same shape, in terms of $E$ vs. $t$ or of $j$ vs. $E$, and appear as those of 'reversible processes': they are the so-called diffusion-controlled charge-transfer processes.

On the other hand, the responses are different from one another whenever the charge transfer is slower than diffusion: a variety of responses, depending on the individual kinetic parameters, are obtained. For non-reversible processes, in fact, a number of different situations are found, since the rate of the charge transfer depends on $E$, increasing at increasing $E$. While at low overvoltages, the charge transfer constitutes the slower step, and the rate of diffusion becomes limiting when high enough overvoltages are applied. In other words, the rate of the same process progressively shifts from being controlled by both charge transfer and diffusion, to be only diffusion-controlled. This always occurs in correspondence to the limiting current.

\section{A quick look at controlled potential techniques}

It seems spontaneous to introduce here a first controlled potential technique that measures the current flowing at an electrode polarised at a constant potential after stepping the potential from a value at which no electrode process is active: the chronoamperometry. As already underlined, our aim is not to furnish details about this and other controlled potential techniques, but only to better exemplify what discussed about mass transport coupled to electrode kinetics in conditioning the relevant responses, specifically with respect to the reversible or non-reversible character.

Figure 6 shows the typical potential waveform for chronoamperometry, together with a few concentration profiles for Red electroactive species, along the unique diffusion direction, $x$. Semi-infinite linear diffusion conditions are assumed to be operative. In the case of Fig. 6, the potential is 'suddenly' stepped from $E_{1}$ to $E_{2}$, corresponding to the already mentioned 'limiting current: the concentration of Red at the electrode is 0 for any $t>0$, so that Eq. (18) expresses the concentration profiles for different $t$ values. It is evident from Fig. $6 \mathrm{~b}$ that the gradient of the electroactive species at the electrode progressively decreases, and the diffusion layer thickness concomitantly increases. No assumption is made about the reversibility degree. The following equation holds:

$j(t)=n F C_{\mathrm{Red}}^{*} D_{\mathrm{Red}}^{1 / 2} \pi^{-1 / 2} t^{-1 / 2}$

Equation (24) is the so-called the Cottrell equation. The general expression, valid for any potential in the case of a reversible process, is: 

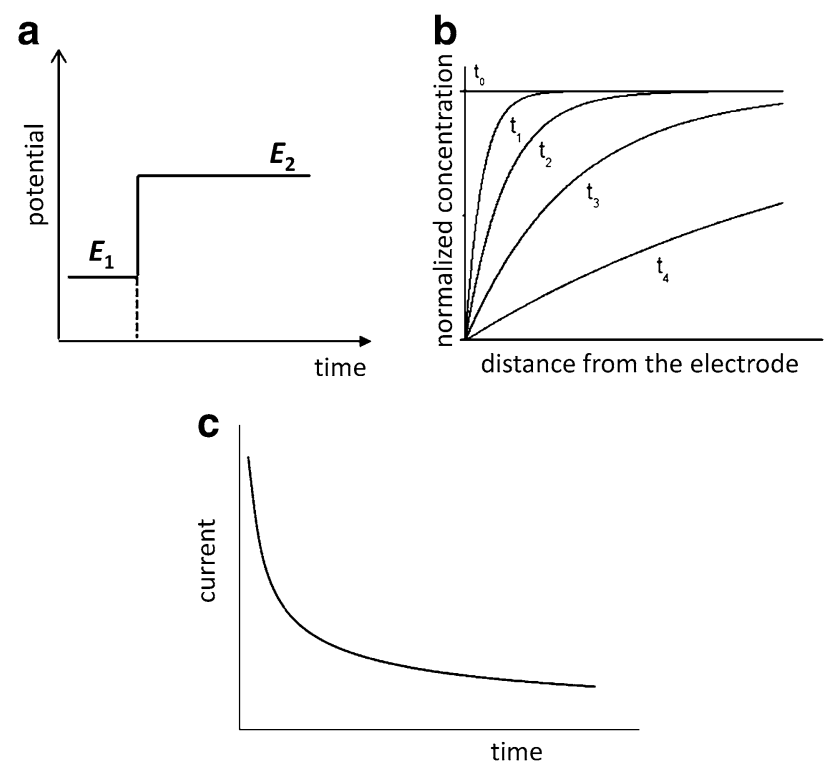

Fig. 6 Chronoamperometry at a potential corresponding to $c_{\mathrm{Red}}(0$; $t)=0$ : a potential waveform; $\mathbf{b} c_{\text {Red }}$ concentration profiles at different times [Eq. (16)]; and $\mathbf{c}$ sketch of a typical signal recorded. Normalization in $\mathbf{b}$ is performed by dividing $c_{\operatorname{Red}}(x ; t)$ by $c_{\mathrm{Red}}^{*}$. Reprinted with permission from Ref. [12]

$j(t)=\frac{n F D_{\mathrm{Red}}^{1 / 2}}{\pi^{1 / 2} t^{1 / 2}(1+\xi \Theta)}$

where

$\xi=\left(\frac{D_{\text {Red }}}{D_{\text {Ox }}}\right)^{1 / 2}$

and

$\Theta=\exp \left[\frac{n F}{R T}\left(E^{0^{\prime}}-E_{2}\right)\right]=\frac{C_{\mathrm{Red}}(0 ; t)}{C_{\mathrm{Ox}}(0 ; t)}$

The dependence of the current on the square root of time, as expressed by Eqs. (24) and (25), accounts for the occurrence of a diffusion-controlled charge-transfer process: the diffusion constitutes the rate limiting step of the whole process, the charge transfer being much faster. This is the reason why, in the above-reported equations, no kinetic parameter is included: the expressions of the current for reversible processes only make use of Fick's laws of diffusion.

The Cottrell equation does not consider the differential capacitive current that has been claimed to couple to the faradic one whenever changes in the electrode potentials are operative. Proper geometry of the electrochemical cell, as well as low electrode capacitance and solution resistance, makes it falls to zero after a time as short as few tens of milliseconds after the imposition of $E_{2}$.

Figure 6c shows a typical chronoamperometric response, as predicted by the Cottrell equation.
Despite the popularity of Eq. (24), Eq. (25) deserves particular attention when considering the validity of the thermodynamic Nernst equation in the frame of a process in which flow of current does occur. As specified above, Eqs. $(24,25)$ hold along the whole time interval explored, at whatever potential value, when the charge transfer is reversible in nature. However, reversibility requires that the values of the concentrations at the electrode fit those predicted by Nernst's equation in a time as short as possible. The shorter the time required to achieve these values, the more reversible the charge transfer, so that the predicted values may not be reached within the time duration of the experiment for high irreversibility of the system. Cottrell equation is obeyed only since the time at which the values predicted by the Nernst equation are reached onwards. The shorter the sampling time at which Nernst equation is first obeyed, the higher the conditional reversibility degree. Noteworthy, factors additional to nonreversibility should be considered at very short times.

By considering voltammetric techniques, CSV can be considered strictly related to normal pulse polarography; however, using a solid electrode is particularly helpful to understand the interrelationships occurring between reversibility degree and experimental conditions. Moreover, it is also suitable to draw experimentally a current vs. potential curve. In these techniques, the electrode is subsequently polarised, in short duration pulses, at increasing potential values; two subsequent pulses differ of a few millivolts. The time duration of each pulse is typically of a few hundreds of seconds. The circuit is open between two subsequent pulses, and different experimental conditions are adopted to reestablish $c_{\mathrm{Ox}}$ and $c_{\mathrm{Red}}$ initial concentrations along the whole diffusion layer, in order that each pulse is applied under the conditions: $c_{\mathrm{Ox}}(0 ; 0)=c_{\mathrm{Ox}}{ }^{*}$ and $c_{\text {Red }}(0 ; 0)=c_{\text {Red }}{ }^{*}$. At each pulse, the current is sampled at a given time and the current vs. potential curve consists of a sequence of points, which may eventually interpolated or regressed to trace a continuous voltammetric curves. It is evident that, for the arguments discussed for chronoamperometry, the sampling time may play a fundamental role: the shorter the time, the higher the reversibility degree requested to the charge transfer in order that the $j$ vs. $E$ curve is typical for a reversible one. On the other hand, at high enough potential, the $\phi_{\mathrm{M}}-\phi_{\mathrm{s}}$ value is high enough to make in all cases the rate of the charge transfer so high to force $c_{\text {Red }}(0 ; t)$ to 0 , whatever the sampling time is. The response accounts for a reversible charge transfer at the potentials of the limiting current: the charge-transfer step is in no cases the rate limiting one.

Actually, since the increase in the potential makes the charge-transfer step faster and faster, it is clear that the conditional reversibility degree exhibited by the response for a non-reversible process even changes in the potential 
interval in which the current increases, before reaching the limiting value.

Figure 7 accounts for different current vs. potential curves for the same process for different sampling times, implying, however, the same conditional reversibility.

A further typical example of different possible time windows over which a charge-transfer process is observed is met with when linear changes of the potential are applied, namely, in linear sweep voltammetry (LSV) and cyclic voltammetry (CV) [14]. In this case, at increasing the potential sweep rate, i.e. at lowering the time given to the system to reach equilibrium, a charge-transfer process may exhibit a progressive transition of the relevant responses from those typical of a reversible to those of a non-reversible process.
Because of these characteristics, LSV and CV are the most suitable techniques to evidence different conditional reversibility degrees of the charge-transfer process. In LSV, the linear potential scan is limited to one single direction (see Fig. 8a). As it is well evident in Fig. 8b, CV is the corresponding 'reversal technique': a backward (reverse) scan follows the forward (direct) one. In Fig. 8c, some representative concentration profiles for the Red electroactive species, at different potential values of the forward scan, are reported for the case of a reversible charge transfer. The LSV response only consists of the $j$ vs. $E$ curve relative to the upper trace of the $\mathrm{CV}$ response in Fig. 8d. The $j$ and $E$ points of the curve that are most significant for both analytical and mechanistic purposes are also indicated in Fig. 8d.
Fig. 7 a Plots showing the collected current vs. time transients and $\mathbf{b}$ the relevant SCV curves constructed from currents recorded at times $\tau_{1}, \tau_{2}$ $\tau_{3}$, and $\tau_{4}$. Reprinted with permission from Ref. [13]
Fig. 8 Potential waveform for a LSV and b CV. c A few concentration profiles for the Red electroactive species in the case of a reversible charge transfer (forward potential scan) are shown. $\mathbf{d}$ CV curve with meaningful current and potential quantities indicated. Noteworthy, the highest value for the gradient at the electrode, corresponding to the maximum in the response in $\mathbf{d}$, occurs at a potential at which $c_{\text {Red }}(x=0)>0$. Reprinted with permission from Ref. [12] a
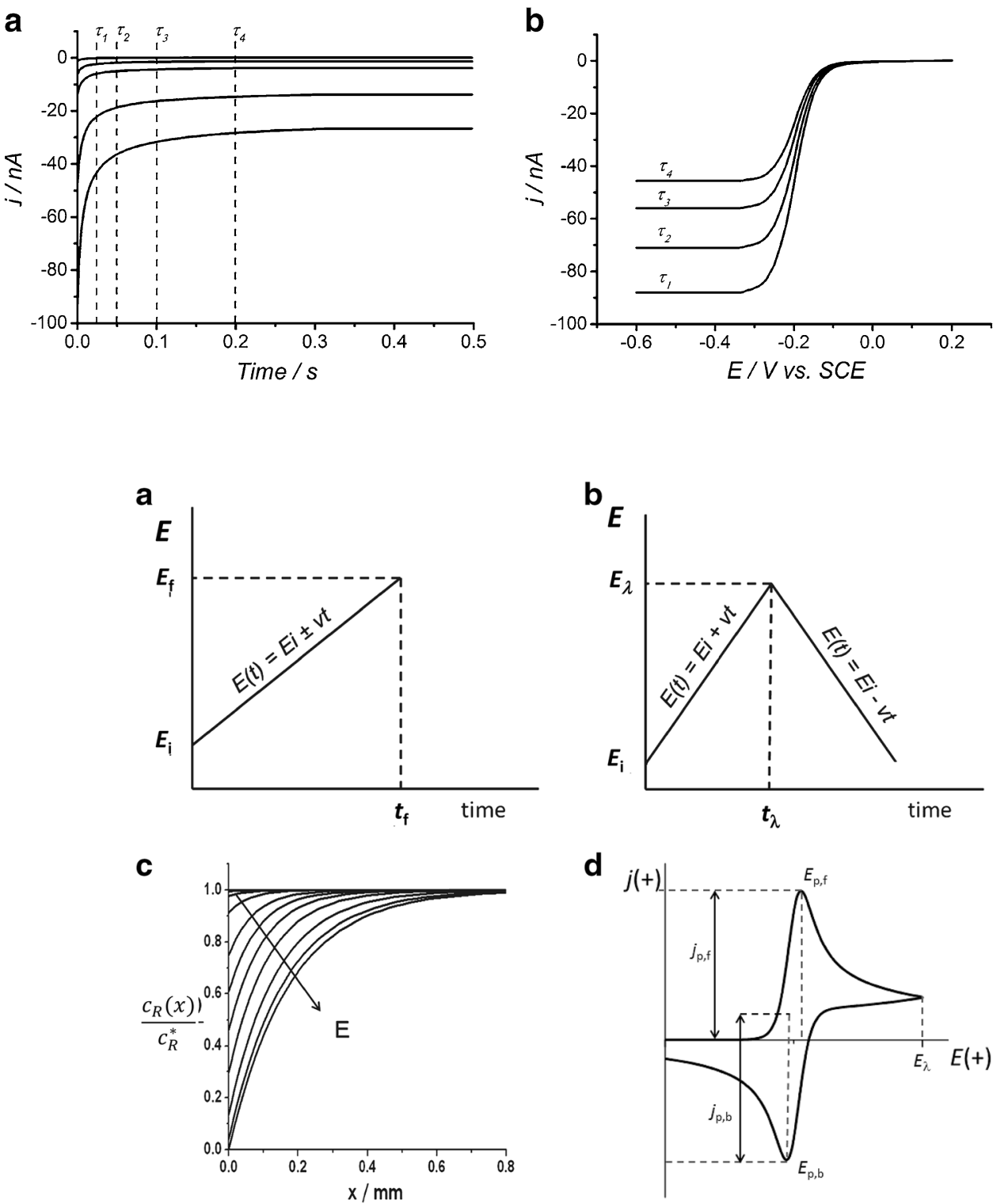

d

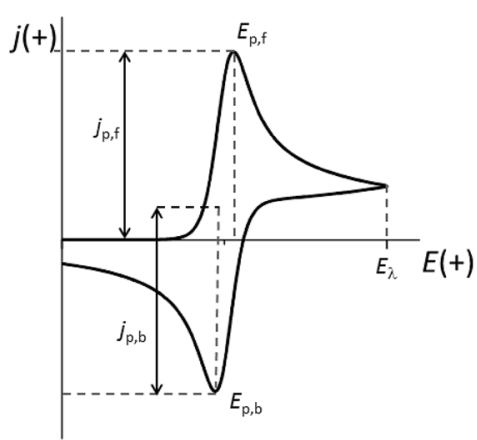


In LSV, the evolution of the concentration profiles for the electroactive species is affected by two counteracting factors: at increasing the potential, the concentration of the reacting species at the electrode progressively decreases either for thermodynamic or for kinetic reasons (think of Nernst or at EGV equations, respectively). This decrease, on its own, makes the gradient at the electrode increase. On the other hand, the perturbation arising at the electrode extends into the solution, lowering the gradient of the Red electroactive species (think of the evolution of the concentration profiles in chronoamperometry-Fig. 6b). The consequent lowering of the relevant flux at the electrode causes, on its own, a decrease in the current density for both reversible and non-reversible charge-transfer processes. As a result, the response exhibits a relative maximum before and after which the former or the latter effect prevails, respectively.

In the backward scan of a CV, over a first potential range, the current still has the same (positive) sign, due to the higher value of the forward with respect to the backward redox reaction. Past this value, the reverse redox process, due to the reduction of the species formed in the forward scan in the proximity of the electrode, assumes progressively increasing prominence: the net current flows in the opposite direction, and a backward (cathodic) response is recorded. The shape of the LSV and of the CV responses allows qualitative and quantitative conclusions about the nature of the electrode charge transfer, i.e. about the relevant reversibility degree. This also holds for the parameters defining more complex mechanisms, in which homogeneous or heterogeneous events are coupled to the charge transfer.

The expressions accounting for the peak current for an uncomplicated reversible charge transfer:

$\left(j_{p}\right)_{\mathrm{rev}}=2.688 \cdot 10^{8} n^{3 / 2} D_{\mathrm{Red}}^{1 / 2} c_{\mathrm{Red}}^{*}\left(\frac{\partial E}{\partial t}\right)^{1 / 2}$

and for an uncomplicated totally irreversible charge transfer:

$\left(j_{p}\right)_{\text {irrev }}=2.987 \cdot 10^{8} \propto^{1 / 2} n^{3 / 2} D_{\operatorname{Red}}^{1 / 2} c_{\operatorname{Red}}^{*}\left(\frac{\partial E}{\partial t}\right)^{1 / 2}$

show that in both cases, a dependence of $j_{\mathrm{p}}$ on $\left(\frac{\partial E}{\partial t}\right)^{1 / 2}$ is exhibited. Noteworthy, a process is often claimed to be 'diffusion controlled' when it exhibits LSV responses with peak current intensity linearly dependent on the square root of the potential scan rate, which is actually not true, in view of Eq. (29). Figure 9 shows that the quasi-reversible charge-transfer processes do not obey such a trend, since increase in the scan rate implies progressive decrease in the reversibility degree, according to what discussed above.

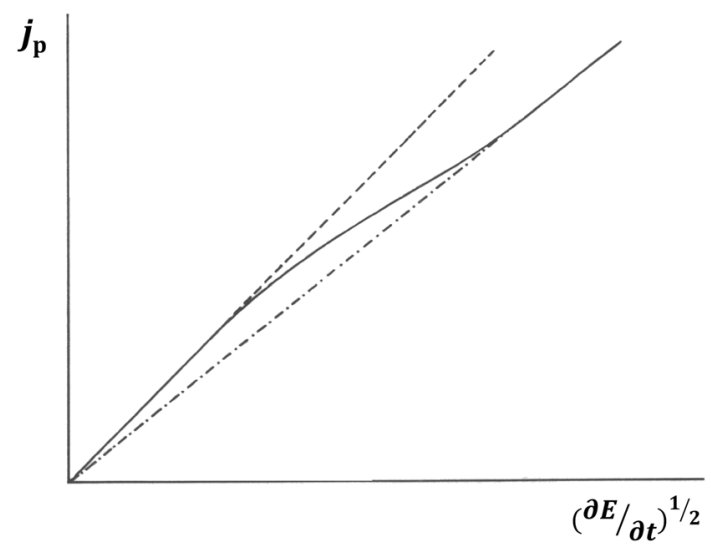

Fig. 9 Plot of $j_{\mathrm{p}}$ vs. $\left(\frac{\partial E}{\partial t}\right)^{1 / 2}$ for a quasi-reversible charge-transfer process, where transition from a reversible to a totally irreversible behaviour is observed (dotted and broken lines, respectively). Reprinted with permission from Ref. [14]

A possible distinction of quasi-reversible from totally irreversible processes is based on the impossibility to define, in the latter case, a finite width potential region in which the response is conditioned significantly by both the forward (oxidation) charge transfer and the backward (reduction) charge transfer. The dimensionless parameter, $\Psi$ :

$\psi=\frac{\left(D_{\mathrm{Red}} / D_{\mathrm{Ox}}\right)^{\alpha / 2} k_{\mathrm{s}, \mathrm{h}}}{\left[D_{\mathrm{Red}}^{1 / 2}(n F / R T)^{1 / 2} \pi\left(\frac{\partial E}{\partial t}\right)^{1 / 2}\right]}$

identifies a family of responses, accounting for (1) the intrinsic reversibility degree of the charge transfer, as defined by $k_{\mathrm{s}, \mathrm{h}}$ and $\alpha$; (2) the time window, approximately defined as the time necessary to cross the whole LSV response, directly related to the potential sweep rate, $\partial E / \partial t$; and (3) the diffusion rate of the electroactive species, as defined by the relevant diffusion coefficient, $D_{\text {Red }}$ and $\mathrm{D}_{\text {ox }}$. $\Psi$ gives a quantitative estimate of the conditional reversibility degree of the charge-transfer process as a whole ${ }^{4}$ in agreement with the discussion reported above as to the factors affecting it. A given response accounts for a

\footnotetext{
${ }^{4}$ A digital simulation technique has been adopted to calculate the curves reported in Supplementary Material. Noteworthy, the equations accounting for the values of the significant quantities of responses relative to $\mathrm{LSV}$ and $\mathrm{CV}$, as well to other controlled potential techniques, reported in the text and drawn out from the cited literature, have been originally computed by solving the system of equations representing the so-called boundary value problem, some examples of which are given in this study. Through the Laplace transform method, the differential equations are converted into a proper dimensionless integral equation. Different analytical, numerical, series methods have been proposed for its solution. Among the dimensionless parameters that have been defined to achieve solution allow to draw the equation for the current/potential curves, the dimensionless parameter $\Psi$ is introduced, as defined in Eq. (27).
} 
number of different situations sharing the value of the dimensionless parameter $\Psi$.

In other words, a family of responses with increasing conditional reversibility, i.e. increasing $\Psi$ values, accounts for a number of processes with increasing $k_{\mathrm{s}, \mathrm{h}}$ values and/or recorded at decreasing potential scan rates $\left(\frac{\partial E}{\partial t}\right)$ and/or for decreasing rate for mass transfer, as accounted for by $D_{\text {Red }}$. Similarly, $\Psi$ assumes a constant value, leading to one single response accounting for a number of intrinsically differently reversible charge transfers, once the potential scan rate progressively changes and/or the diffusion rate changes as well. Typically, it will be very difficult, or even impossible, to realise a combination of the factors leading to the definition of $\Psi$, i.e. of the intrinsic character of the charge-transfer process and of the experimental variables, so that responses shifting from those typical for a reversible to those for a totally irreversible process are recorded. According to the literature, responses characteristic of a reversible charge-transfer processes is obtained for $\Psi>7$, while responses typical of totally irreversible processes are recorded for $\Psi<10^{-3}$.

In the file available as Supplementary Material, CV responses for uncomplicated charge transfers with different reversibility degree are shown, together with the concentration profiles of reactant and product at some selected potentials along the forward and the backward sweeps.

Since LSV and CV are the most widely used voltammetric techniques to study and even quantify the kinetic parameters characterising an electrode process, we insert here a discussion of the quantities defining the relevant responses, for an uncomplicated electrode charge transfer, at varying the reversibility degree [15-17]. In addition to the peak current densities, as expressed by Eqs. (28) and (29), other typical quantities, referred to the potential scale, distinguish LSV and CV responses for reversible from totally irreversible processes. For a reversible response:

$E_{\mathrm{p}, \mathrm{f}}-E_{\mathrm{p} / 2, \mathrm{f}}=2.199 \frac{R T}{n F}\left(=56.5 / n \mathrm{mV}\right.$ at $\left.25^{\circ} \mathrm{C}\right)$

where $E_{\mathrm{p}, \mathrm{f}}$ and $E_{\mathrm{p} / 2, \mathrm{f}}$ are the potential corresponding to the peak of the forward scan and to the half the current peak, respectively, and

$E_{\mathrm{p}, \mathrm{f}}-E_{\mathrm{p}, \mathrm{b}}=2.218 \frac{R T}{n F}\left(=57 / n \mathrm{mV}\right.$ at $\left.25^{\circ} \mathrm{C}\right)$

Moreover, the ratio between the backward and forward peak currents for a reversible response tends to unity once the baseline for the backward peak is properly computed [14]. The unity value and the $57 / \mathrm{n} \mathrm{mV}$ for peak-to-peak separation are actually asymptotic values, reached by switching the potential scan direction at more and more positive values. At increasing the non-reversible character of the process, the relevant peak-shaped responses exhibit progressive broadening, implying higher and higher values of the potential differences given above. As a consequence of the further and further location of the backward and forward peaks, the ratio between current peak values progressively decreases, due to the time elapsed between generation of Ox species and polarisation of the electrode at potentials at which it is reduced back to Red. As an example, the values for $\left(E_{\mathrm{p}, \mathrm{f}}-E_{\mathrm{p}, \mathrm{b}}\right) \times n$ have been computed to $63 \mathrm{mV}$ for $\Psi=7$, to $84 \mathrm{mV}$ for $\Psi=1$, and to $212 \mathrm{mV}$ for $\Psi=0.1$ [17]

A further example of changes of the conditional reversibility degree of the charge transfer, as evident from the relevant voltammetric responses, may be given by a process occurring at an $\operatorname{RDE}[11,18-20]$. If the electrode is disc-shaped, embedded in a rod of an insulating material and rotating with an angular velocity $\omega_{\mathrm{r}}$, the axis of rotation passes through the centre of the disc and results perpendicular to the surface: the diffusion layer thickness, $\delta$, accounts for the concentration gradient in the $x$-direction.

The diffusion layer thickness is a crucial parameter in the diffusion equations for RDE. It accounts for the distance from the electrode surface at which no hydrodynamic motion of the solution is assumed to occur: the mass transport takes place by molecular mechanism, mostly by diffusion. The exact solution of the respective convectivediffusion equations is very complicated.

In the case of a laminar flow, the flow velocity is zero at the plane electrode surface, then continuously increases within a given layer (the Prandtl boundary layer), and eventually reaches the value characteristic to the stirred liquid phase.

The convective-diffusion equations have been solved for RDE:

$\delta_{\operatorname{Red}}=1.61 D_{\operatorname{Red}}^{1 / 3} \omega^{-1 / 2} v^{1 / 6}$

$j=0.62 n F D_{\mathrm{Red}}^{2 / 3} \omega^{1 / 2} v^{1 / 6}\left[c_{\mathrm{Red}}(x=0)-c_{\mathrm{Red}}^{*}\right]$

where $v$ is the kinematic viscosity. When $c_{\text {Red }}(x=0)=0$, i.e. $j=j_{\mathrm{L}}$, Eq. (34) is called the Levich equation.

The higher the rotation speed, $\omega$, the higher the mass transport rate to and from the electrode surface, the higher the flux of the electroactive species at the electrode, and the higher the current. High charge-transfer rates are also required to possibly achieve Nernstian equilibrium.

By operatively defining a 'mass transfer constant', $k_{\mathrm{m}, \text { Red }}$, related to the rotation speed and, hence, to what this implies as to mass transfer rate of the electroactive reactant species, a comparison is possible with the charge-transfer rate, as expressed by the standard heterogeneous chargetransfer constant, $k_{\mathrm{s}, \mathrm{h}}$, or by the exchange current density, $j_{0}$. The plot in Fig. 10 shows how a given process may lead to responses with different conditional reversibility degrees and, at the same time, how charge transfers with actually 


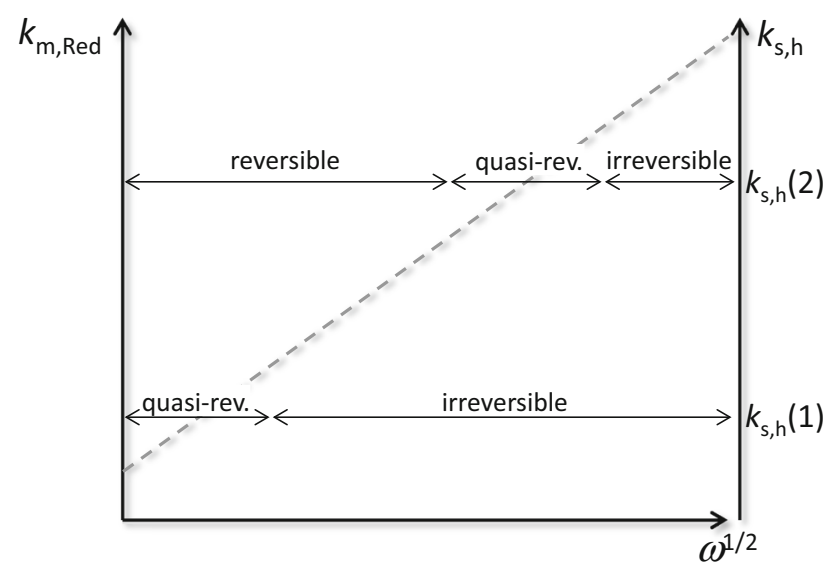

Fig. 10 The same as in Fig. 2 for the case of an RDE; the time window $\tau^{-1}$ is accounted for by $\omega^{1 / 2}$

different reversibility degrees may exhibit similar responses.

The standard rate constants $\left[k_{\mathrm{s}, \mathrm{h}}(1)\right.$ and $\left.k_{\mathrm{s}, \mathrm{h}}(2)\right]$ are the characteristics to the charge-transfer rate of the given systems. The diffusion rate constants $k_{\mathrm{m} \text {, Red }}$ are varied by the rotation rate of the electrode. Two systems with different electron-transfer rates $\left[k_{\mathrm{s}, \mathrm{h}}\right.$ (1) and $\left.k_{\mathrm{s}, \mathrm{h}}(2)\right]$ are considered. If $k_{\mathrm{s}, \mathrm{h}} \gg k_{\mathrm{m} \text {, Red }}$ the system is reversible, while in the case of $k_{\mathrm{m}, \operatorname{Red}} \gg k_{\mathrm{s}, \mathrm{h}}$, irreversible behaviour is observed. The values of the diffusion coefficients are taken equal for both systems. Adapted from Ref. [11].

Figure 11 shows different RDE voltammetric curves relative to different charge-transfer processes. By decreasing the conditional reversibility degree, either by increasing the rotation speed $\omega$ or by decreasing $k_{\mathrm{s}, \mathrm{h}}$, the current lowers and the charge transfer overvoltage shifts the response to higher potentials. The different shapes of

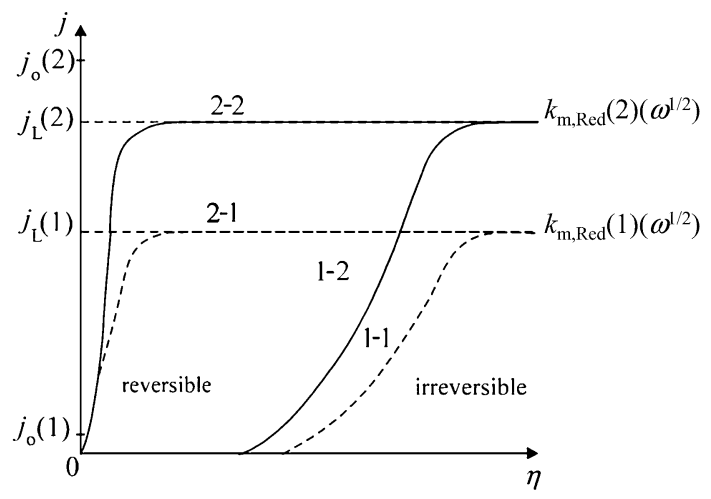

Fig. 11 Steady-state polarization curves $\left(j\right.$ vs. $\eta$ ) for a reversible $\left(j_{0}\right.$ (2)) and an irreversible $\left(j_{0}(1)\right)$ anodic process, respectively, at two different stirring rates. $j_{\mathrm{L}}$ represents the limiting current. The following parameters refer to the different curves: $j_{0}(1)$ and $k_{\mathrm{m} \text {,Red }}$ (1) (curve $1-1$ ); $j_{0}(1)$ and $k_{\mathrm{m}, \text { Red }}(2)$ (curve $\left.1-2\right) ; j_{0}(2)$ and $k_{\mathrm{m}, \text { Red }}(1)$ (curve 2-1) and $j_{0}(2)$ and $k_{\mathrm{m}, \text { Red }}(2)$ (curve 2-2); The same diffusion coefficients for both systems are considered. Adapted from Ref. [11] the responses are also evidenced, as well as the dependence of the mass transfer rate, as expressed by the concentration of electroactive species reacting at the electrode, on $\omega^{1 / 2}$ or by $j_{0}$, or by a combination of the two parameters; this is the case of a mixed control, i.e. of quasi-reversible chargetransfer processes. The limiting current values do not depend on the reversibility degree of the charge transfer, but depend on the RDE rotation speed.

Figure 12a shows the directions along which the rotation of the RDE induces mass transport by convection. Turbulent flow activates uncontrolled convection and should be minimised: laminar flow is given account in Fig. 12a. Figure 12b shows the concentration profiles of the electroactive species at different rotation rates. The $j$ vs. $E$ curves assume sigmoidal shapes, exhibiting the already cited limiting current, at high enough potentials: in the case of reversible processes, Nernst equation imposes $c_{\text {Red }}(0$; $t)=0$ and, in the cases of non-reversible charge transfers, $c_{\text {Red }}(0 ; t)=0$ is a consequence of high enough overvoltages, imposing a charge-transfer rate much faster than diffusion.

\section{Boundary value problem for different reversibility degrees}

As above cited, whenever the value of the electrode potential is controlled according to a given dependence on time, the so-called potential waveform defines the relevant controlled potential technique: the potential applied to WE is either fixed or differently varied over time. Two cases are defined, in which (1) the computation of the current density comes out only from the concentration profiles of the species involved in the electron-transfer process, hence, on the concentration gradient at the electrode surface, or (2) the concentration gradient is 'filtered' by the EGV equation. In the reversible case, every single Red entity that arrives at the electrode reacts or not only in dependence of the requirements of the Nernst equation, and EGV equation does not play any role. In a non-reversible charge-transfer case, the flux conditions the Red concentration at the electrode that reacts to an extent conditioned by EGV kinetic equation. In the latter case, a number of situations are possible, in which the rate of the mass transfer plays anyway a role.

For an uncomplicated charge transfer, in which both Red and Ox species are soluble and stable in solution, a system of differential equations of parabolic type, consisting of the expressions of Fick's second law referred to Red and Ox species [Eqs. (22) and (23)], should be solved under proper boundary conditions. These differ in part from one another in dependence of the reversibility degree of the charge transfer. The whole of the differential 

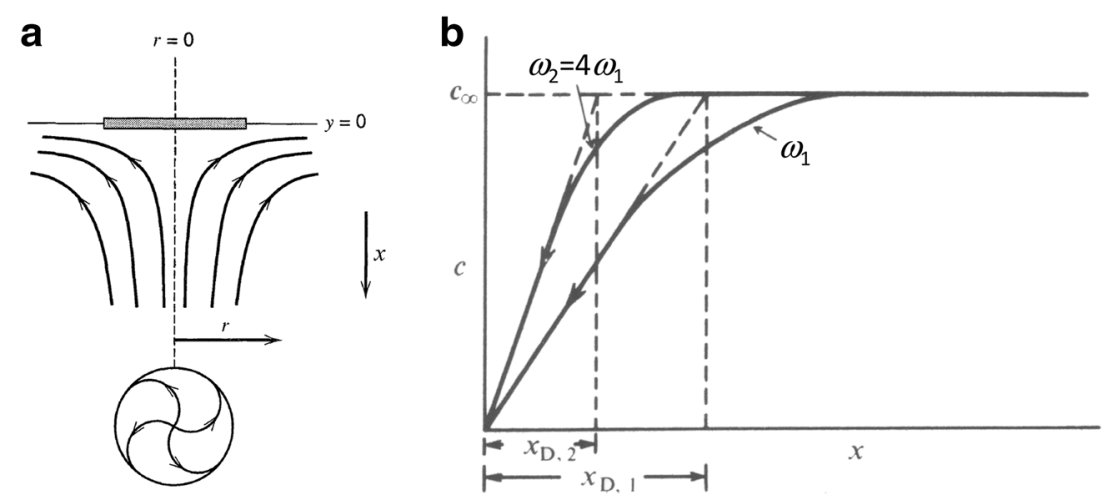

Fig. 12 a Schematic representation of the flux lines for an RDE, under laminar flow conditions; turbulent flow is minimised by suitable experimental setup and conditions reproduced with permission from Ref. [8]. b Concentration profiles for the electroactive species, along the direction orthogonal to the electrode surface ( $x$ direction) at two different rotation speeds $-x_{\mathrm{D}, 1-2}$ represent the Nernst diffusion layers, where both diffusion and convection mass transports occur. Reproduced with permission from Ref. [19]

$$
\operatorname{Red}^{\mathrm{z}} \leftrightarrows 0 \mathrm{x}^{\mathrm{z} \prime}+n \mathrm{e}
$$

$\frac{\partial c_{\text {Red }}}{\partial t}=D_{\operatorname{Red}} \frac{\partial^{2} c_{\operatorname{Red}}(x ; t)}{\partial x^{2}}$

initial condition:

$t=0$
$x \geq 0$$\left\{\begin{array}{l}c_{\mathrm{Red}}(x ; 0)=c_{\mathrm{Red}}^{*} \\ c_{\mathrm{Ox}}(x ; 0)=c_{\mathrm{Ox}}^{*}\end{array}\right.$

Boundary conditions:

$t>0$
$x \rightarrow \infty$$\left\{\begin{array}{l}c_{\mathrm{Red}}(x ; 0)=c_{\mathrm{Red}}^{*} \\ c_{\mathrm{Ox}}(x ; 0)=c_{\mathrm{Ox}}^{*}\end{array}\right.$

$t>0$
$x=0$$\left\{\begin{array}{l}D_{\mathrm{Red}} \frac{\partial c_{\mathrm{Red}}(0 ; t)}{\partial x}=-D_{\mathrm{Ox}} \frac{\partial c_{\mathrm{OX}}(0 ; t)}{\partial x}\left(=\frac{j(t)}{\mathrm{n} F}\right) \\ \frac{c_{\mathrm{Red}}(0 ; t)}{c_{\mathrm{OX}}(0 ; t)}=\exp \left[\frac{\mathrm{n} F}{R T}\left(E(t)-E_{O x / \text { Red }}^{\theta \prime}\right)\right]\end{array}\right.$

Scheme 1 Boundary value problem in the case of reversible charge transfer for uncomplicated electrode processes

equations and the proper boundary conditions is often indicated as boundary value problem.

Schemes 1, 2 and 3 show the boundary value problems for reversible, quasi-reversible, and totally irreversible charge transfers, respectively. The 'quasi-reversible' denomination indicates a charge transfer, which, though non-reversible, is characterised, over a finite potential range, by significantly different from zero heterogeneous kinetic constants for both oxidation and reduction (forward

$$
\operatorname{Red}^{\mathrm{z}} \stackrel{k_{\mathrm{s}, \mathrm{h}} ; \alpha}{\rightarrow} \mathbf{O x}^{\mathrm{z} \prime}+\boldsymbol{n e}
$$

$\frac{\partial c_{\operatorname{Red}}}{\partial t}=D_{\operatorname{Red}} \frac{\partial^{2} c_{\operatorname{Red}}(x ; t)}{\partial x^{2}}$

$\frac{\partial c_{0 \mathrm{X}}}{\partial t}=D_{\mathrm{Ox}} \frac{\partial^{2} c_{O X}(x ; t)}{\partial x^{2}}$

initial condition:

$t=0$
$x \geq 0$$\left\{\begin{array}{l}c_{\mathrm{Red}}(x ; 0)=c_{\mathrm{Red}}^{*} \\ c_{\mathrm{Ox}}(x ; 0)=c_{\mathrm{Ox}}^{*}\end{array}\right.$

boundary conditions:

$t>0 \quad\left\{\begin{array}{l}c_{\mathrm{Red}}(x ; 0)=c_{\mathrm{Red}}^{*} \\ c_{\mathrm{Ox}}(x ; 0)=c_{\mathrm{Ox}}^{*}\end{array}\right.$

$x=0 \quad \begin{cases}D_{\mathrm{Red}} \frac{\partial c_{\mathrm{Red}}(0 ; t)}{\partial x}=-D_{\mathrm{Ox}} \frac{\partial c_{\mathrm{OX}}(0 ; t)}{\partial x} & \left(=\frac{j(t)}{n F}\right) \\ D_{\mathrm{Red}} \frac{\partial c_{\mathrm{Red}}(0 ; t)}{\partial x}=k_{\mathrm{f}, \mathrm{h}} c_{\mathrm{Red}}(0 ; t) & \left(=\frac{j(t)}{n F}\right)\end{cases}$

( $\eta$ in $k_{\mathrm{f}, \mathrm{h}}$ is referred to $E^{\theta^{\prime}}$ )

Scheme 2 Boundary value problem in the case of totally irreversible charge transfers for uncomplicated electrode processes

and backward charge transfers, respectively). On the contrary, a similar range is not identifiable for totally charge transfers: either the forward or the backward kinetic rate constant is only significantly different for 0 . Noteworthy, the term initial conditions is often used for the $t=0$ boundary.

The cases of chemical reactions coupled to the charge transfer are not considered here, though being very 


\section{$k_{\mathrm{s}, \mathrm{h}} ; \alpha$ \\ $\operatorname{Red}^{\mathrm{z}} \stackrel{\mathbf{O x}^{\mathrm{z} \prime}}{\leftrightarrows}+\mathbf{n e}$}

$\frac{\partial c_{\mathrm{Red}}}{\partial t}=D_{\mathrm{Red}} \frac{\partial^{2} c_{\mathrm{Red}}(x ; t)}{\partial x^{2}}$

$$
\frac{\partial c_{\mathrm{OX}}}{\partial t}=D_{\mathrm{Ox}} \frac{\partial^{2} c_{O x}(x ; t)}{\partial x^{2}}
$$

initial condition:

$t=0$
$x \geq 0$$\left\{\begin{array}{l}c_{\mathrm{Red}}(x ; 0)=c_{\mathrm{Red}}^{*} \\ c_{\mathrm{Ox}}(x ; 0)=c_{\mathrm{Ox}}^{*}\end{array}\right.$

boundary conditions:

$t>0$
$x \rightarrow \infty$$\left\{\begin{array}{l}c_{\mathrm{Red}}(x ; 0)=c_{\mathrm{Red}}^{*} \\ c_{\mathrm{Ox}}(x ; 0)=c_{\mathrm{Ox}}^{*}\end{array}\right.$

$t>0 \quad\left\{\begin{array}{l}D_{\mathrm{Red}} \frac{\partial c_{\mathrm{Red}}(0 ; t)}{\partial x}=-D_{\mathrm{Ox}} \frac{\partial c_{\mathrm{Ox}}(0 ; t)}{\partial x} \quad\left(=\frac{j(t)}{n F}\right) \\ D_{\mathrm{Red}} \frac{\partial c_{\mathrm{Red}}(0 ; t)}{\partial x}=k_{\mathrm{f}, \mathrm{h}} c_{\mathrm{Red}}(0 ; t)-k_{\mathrm{b}, \mathrm{h}} c_{\mathrm{Ox}}(0 ; t) \quad\left(=\frac{j(t)}{n F}\right)\end{array}\right.$

( $\eta$ in $k_{\mathrm{b}, \mathrm{h}}$ is also referred to $E^{\theta^{\prime}}$ )

Scheme 3 Boundary value problem in the case of quasi-reversible charge transfers for uncomplicated electrode processes

important in studies of molecular electrochemistry; the concentration changes due to the events additional to diffusion should be accounted for, integrating the II Fick's law constituting the differential equations in the cases presented here.

Different approaches have been used to solve the variety of systems of differential equations: from semianalytical methods, in which the analytical mathematical procedure is complemented by numerical techniques, to fully numerical ones. The so-called digital simulation techniques have been mainly used for a long time [21]. In these techniques, the time and the space interested by diffusion are differently discretised through the so-called space-time grid, dense enough to account properly for the concentration changes induced by the charge transfer and by the diffusion.

\section{References}

1. Inzelt $\mathrm{G}$ (2014) Crossing the bridge between thermodynamics and electrochemistry. From the potential of the cell reaction to the electrode potential. ChemTexts 1(2):1-11

2. Seeber R, Zanardi C, Inzelt G (2015) Links between electrochemical thermodynamics and kinetics. ChemTexts 1:1-16

3. Denbigh K (1981) The principles of chemical equilibrium, IV edn. University Press, Cambridge

4. Southampton Electrochemistry Group (1985) Instrumental methods in electrochemistry. Ellis Horwood Limited, Cambridge

5. Gileadi E (1993) Electrode kinetics. VCH, New York

6. Brett CMA, Oliveira Brett AM (1993) Electrochemistry: principles, methods, and applications. Oxford University Press, Oxford

7. Oldham HB, Myland JC (1994) Fundamentals of electrochemical science. Academic Press, San Diego

8. Bard AJ, Faulkner LR (2001) Electrochemical methods, fundamentals and applications, 2nd edn. Wiley, New York

9. Scholz F (ed) (2002) Electroanalytical methods. Guide to experiments and application. Springer, Berlin, Heidelberg

10. Scholz F (2010) Electroanalytical methods, guide to experiments and applications, 2nd revised and extendededn. Springer, Berlin Heidelberg

11. Inzelt G (2010) Kinetics of electrochemical reactions. In: Scholz F (ed) Electroanalytical methods, guide to experiments and applications, Ch I. 3, 2nd, revised and extended edn. Springer, Berlin, Heidelberg, pp 33-53

12. Moretto LM, Seeber R (2014) In: Moretto LM, Kalcher K (eds) Environmental analysis by electrochemical sensors and biosensors. Fundamentals, vol 1, Ch II 3, Springer, Berlin, Heidelberg, pp 239-282

13. Perry SC, Al Shandoudi LM, Denuault G (2014) Sampled-current voltammetry at microdisk electrodes: kinetic information from pseudo steady state voltammograms. Anal Chem 86:9917-9923

14. Bontempelli G, Magno F, Mazzocchin GA, Seeber R (1989) Linear sweep and cyclic voltammetry. Ann Chim (Rome) 79:103-216

15. Matsuda H, Ayabe Y (1954) Zur Theorie der Randles-Sevčikschen Kathodenstfrahl-Polarographie. Z Elektrochem 59:494-503

16. Nicholson RS, Shain I (1964) Theory of stationary electrode polarography. single scan and cyclic methods applied to reversible, irreversible, and kinetic systems. Anal Chem 36:706-723

17. Nicholson RS (1965) Theory and application of cyclic voltammetry for measurement of electrode reaction kinetics. Anal Chem 37:1351-1355

18. Newman JS (1973) Electrochemical systems. Prentice Hall, Englewood Cliffs

19. Albery WJ (1975) Electrode kinetics. Oxford University Press, Oxford

20. Pleskov YuV, Yu Filinovskii V (1976) The rotating disc electrode. Consultants Bureau, New York

21. Britz D (2005) Digital simulation in electrochemistry, 3rd edn. In: Lecture notes in physics. Springer, Berlin, Heidelberg 